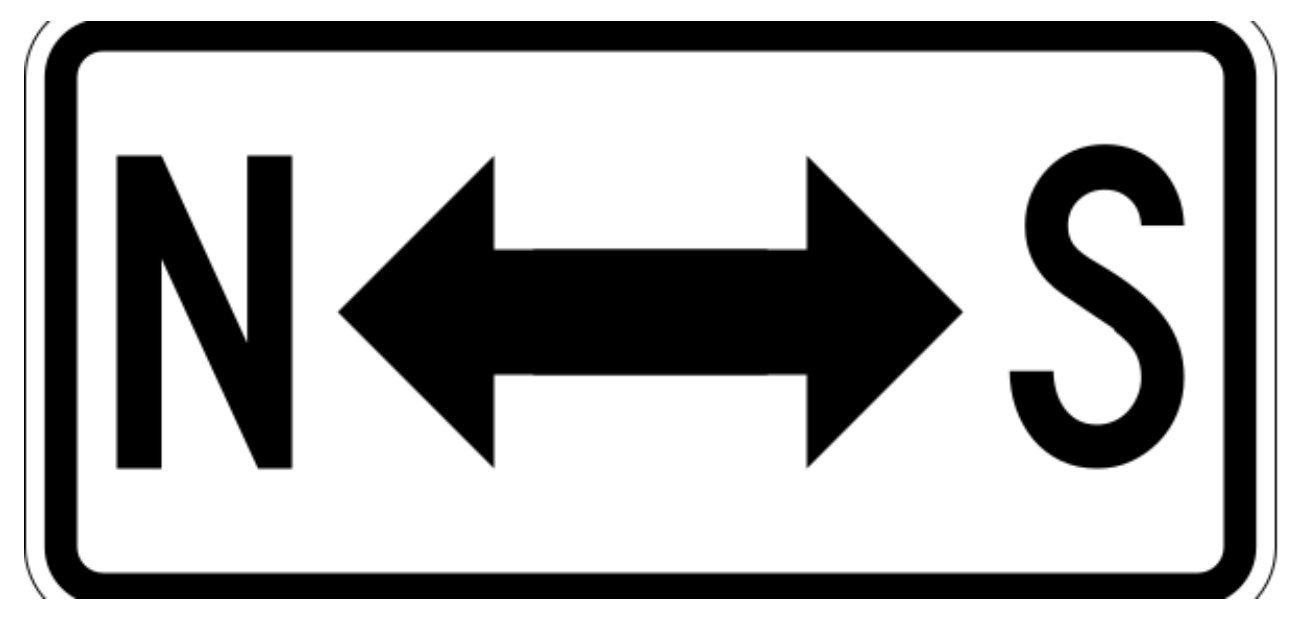

\title{
SETTING THE PRIORITIES RIGHT IN THE COVID-19 PANDEMIC
}

\author{
Carlos Rodriguez ${ }^{1}$
}

1 Fundación Jiménez Díaz

Funding: The author(s) received no specific funding for this work.

Potential competing interests: The author(s) declared that no potential competing interests exist.

\section{Abstract}

When reading the literature (science and media alike) on Covid-19 one get the feeling that people have given up on a medical treatment for the disease. We refer to the virus as something we should avoid at any rate, lest we get infected and die from it. Hence all the clatter about new outbreaks, social distancing, quarantenes and lockdowns. At times, it seems as if we were already counting on a world shutdown until the (hopefully soon) arrival of the much awaited vaccine.

In this paper, I describe a totally opposing view. In my opinion, we are just forgetting that treating this virus should not be such a huge problem, provided that we set the right priorities. If there is a take home message that I want to convey, this is the following: We should aim at preventing death, not infection. With this priority in mind, we will see that the solution follows naturally. This solution is a prophylactic treatment designed only for the people at risk of dying from Covid-19 (elderly and people with comorbidities). The treatment is both antiviral and antiinflammatory, is applied directly where the fire ignites (in the lungs), in the form of an inhaler, and should begin as soon as the first flu-like symptoms show up, not days or weeks later.

My view is that of a world that has an immediate answer to the pandemic. One that provides a treatment to the disease 
and ensures that people won't need to go though ICUs and assited ventilation to safe their lives. One that will not rely on the success or failure in the development of a vaccine for Covid-19.

I will start off by quoting today's news in the US media (2 August 2020): "New CDC forecast projects 20,000 Covid-19 deaths in 21 days". By now, we have been getting so familiar with this kind of headlines that we tend to forget what they imply. What they imply is that we don't have anything in our hands to prevent death in Covid-19 and that our power is just limited to predict the odds of getting severly sick and eventually die.There is nothing to do, except to pray for the best and hope that rather sooner than later we'll get the desperately awaited vaccine.

But, wait a minute. Haven't we already, well into the 21 rst century, the best medical advances ever? Haven't we reached a state of the art medical care, one that is not afraid of dealing with the most complex health challenges like fancy organ transplants, state of the art anti-cancer immune modulators, or biologics of all shapes and colors? Are you telling me that we cannot deal with a virus unless we quickly almost desperately get a vaccine? Let me imagine for a second what future generations will tell their kids about the Covid-19 pandemic: "You know. That was a really bad blow. Millions had to die the worst possible way: suffocating with flooded lungs. Thankfully today we know better. We have this little device that does the trick, so we don't have to worry about a vaccine that, as of today, is still in the waiting". Since this is a possible scenario for the coming future, I will try here to fill the time gap with a different script, one that will hopefully change this sciencefiction story that I just prompted and leave future generations with a brighter account to tell their grandkids about the years of the pandemic.

We need an action plan. But not the one that we have right now. As a kid, I was taught that you cannot put all the eggs in one basket. And this is exactly what I think we are doing with the, otherwise legitimate, hopes for a prompt vaccine. Let me tell you what I think about it: Entrusting everything to this very hypothetical plan A is, to say the least, risky, if not plain irresponsable.

\section{THE “0 DEATHS" MARK FOR COVID-19}

You would surely agree with me that to efficiently combat such a formidable enemy, we should have a clear, ordered set of well defined priorities. In essence, war, pandemics and other catastrophes need to be approached with the same sense of urgency, and, at the same time, with the necessary deliberation and weighing in order to not cause additional harm. As I said, after a proper situation appraisal, we have to define priorities. Not an easy task.

But after all these months watching with a certain distance the pandemic unfold, we should have by now a bit of an advantage to set the priorities right. So, before I discuss with you what we should do, let's start with the easiest part: what we shouldn't.

Here is a list of what I think some decision makers are getting wrong about the way to go with the problem: 


\section{WRONG PRIORITIES}

1. To aim for a universal Covid-19 immunity passport based on seroprevalence

2. To aim at having a lower death rate than your neighbour's ICU.

3. To concentrate the efforts on fighting the virus (i.e. to diminish the viral load) instead of the amok inflammatory process that is the cause of SARS (and consequently of death).

4. To eradicate the disease from the surface of the earth (not a priority right now. Maybe later).

\section{RIGHT PRIORITIES}

A very simple one:

1. To aim for "0 deaths" in the Covid-19 pandemic ( $\Leftrightarrow$ No SARS)

Current policies around the world rely on the following more or less admitted surmise: Let's wait until an effective vaccine is in place. Until then, we will cope with the situation as well as we can, following a general consensus: avoid new socalled outbreaks by testing, tracing and general society-wise social distancing. All of this, while hoping that the treatments in place do the rest, until the messiah/vaccine descends from heaven to save us from hell. Although not openly/officially admitted as such, we all know this is what we call the global plan A, the one that now drives the decisions of most policymakers worldwide, from the WHO to government agencies.

But, with this plan A, aren't we forgetting something? Something that, in any emergency plan should be the obvious number 1 priority? In short, right now, our plan A should be to avoid death. When we'll be close to get to this "O deaths" mark, then, we can talk about the rest: herd immunity, vaccines, or, importantly, long term morbidity in recovered patients. Only imagine for a second that we were able to reach that goal (close to 0 deaths), without the unbearable daily death tolls that we still have as of today, then, it would be much, much easier to think about the whole problem and come up with real solutions to the pandemic. Would you agree with that?

Now, I'll let you ask me a legitimate question: am I not aiming for the sky with this goal? In other words, is that (to aim for the "0 deaths" mark) really possible? Well, that's the reason why I decided to write this one. Because I am 100\% confident that this is not only possible, it's absolutely feasible.

\section{Plan A should focus on preventing death, not infection}

We should start with a single point: An essential part of the plan should be to have people at risk well protected for the eventuality of contracting the disease. After all, Covid-19 would just be another (more or less mild/nasty) coronavirus disease were it not for the Severe Respiratory Distress Syndrome (SARS) that is present in a minority of cases and that is the reason for the deadly hue associated with the disease. So, with the right priorities in mind, we should now start defining the right strategy. We can safely define two obvious arms in this protection strategy: I) Protection from infection, and II) 
Prevention of SARS in people with (a suspected or confirmed) infection.

\section{Protect vulnerable people. Not everybody at any cost}

The first point shouldn't be very difficult to address, let's do social distancing, but, very important, only with people considered at risk of death (you know, elderly and people with comorbidities that haven't been exposed yet to the virus). These people should be protected at any cost. But, let's not paralize entire countries, please! The latter have been happening over the last month especially in countries that were of late recovering from the so-called first wave. Now, the public outcry is over the so-called new outbreaks and the fear of a new death wave. But, and this is the main point of this article, there won't be such a new death wave. Not if we have a true plan. This plan should include, now we know it, an effective treatment to prevent people at risk from ending up in the ICU and eventually die. What! A treatment? Yes, a treatment. A real plan couldn't be called such without a true, effective treatment.

But, as we have just said, there is no such a plan when your main and almost sole bet is a vaccine. As I said, Health Officials worldwide have given up on an non-vaccine effective treatment against the disease and, like a groggy boxer, are just waiting for the bell/vaccine to save their combat.

\section{The global Plan A. Wrong priorities = Wrong plan .}

Let's start with a blunt question: What if we don't get a vaccine in the time frame we hope for? Or, more dramatically put, what if we don't get a vaccine at all? After all, this wouldn't be the first case where too much expectation is put on vaccine development to, at the end of the road (after 30 years in the case of HIV), not having any vaccine at all. Besides, we are all aware of the intrinsic difficulties involved in the development of vaccines for respiratory viruses (RSV ${ }^{[1]}$, just to mention one still in the waiting after decades of huge international efforts $)^{[2]}$. So, despite all the wishful headlines we read every day, that wouldn't be a surprise to be, 5 years down the road, with no vaccine at all.

Second, what if the vaccine does not do the job as expected or, worst, have side effects that make massive vaccination unfeasible [ $^{[3][4][5]}$ Remember, to say the least, this is a very unusual virus from the herd immunity stand point. Just to mention, experts are already quickly changing terms and, realizing that we are probably never going to get more than a $20 \%$ seroprevalence, they are now renaming the good old herd immunity with the new more fashionable name herd immunity threshold (much better suited for the occasion) ${ }^{[6][7][8][9]}$.

Third, in the same vein, what if all the efforts to promote a strong neutralizing antibody response is not the way to go, and all the international massive investments in a traditional vaccine (one driven by the classical antibody response) go down the drain for not considering in time the need to stimulate the so-called cellular immunity or other still unknown immune mechanisms that this strange virus might be invoking? ${ }^{[10][11][12][13][14]}$.

And finally, the best case scenario. Imagine that, in a very optimistic forecast, we have a safe and efficient vaccine by the summer of 2021. Even then, we would still have a one year window with hundreds of thousands, if not millions, of totally 
avoidable deaths. Only because we didn't set the one essential priority right... Wouldn't you agree with that?

\section{Preventing death by the Severe Acute Respiratory Syndrome (SARS). Antivirals, Antiinflammatories or both?}

To properly address this point, let's start with a simple question: Is Covid-19 a viral infection? The simple answer would be, of course, a plain yes. After all, the disease is triggered by a virus, the SARS-CoV2 coronavirus, that infects airway's epithelial cells, causing local tissue damage in the lungs and other tissues, followed by symptoms thereof, and, after raising a more or less robust immune response in the human host, gets resolved (more or less) efficiently. Up to here, it sounds pretty consistent with the view of a classical infection by a respiratory virus. The problem is that, in a minority of cases, (around 10\%, depending on the series), patients develop respiratory failure, what is called SARS, something that has been previously observed with other coronavirus (SARS-CoV1, MERS) as well as with other respiratory viruses like IAV or RSV. SARS has its own physiopathology: it appears in a selected group of people at risk that include older people, or people with comorbidities like cardiovascular diseases or diabetes ${ }^{[15][16]}$. People predisposed to SARS seem to have a higher background level of immune reactivity ${ }^{[17]}$ and in those patients, an uncontrolled immune reaction appears to take hold of the infection, ending with disruption of the endothelial-alveolar barrier ${ }^{[18][19]}$, the occupancy of the alveolar space with liquid and the ensuing respiratory failure. Most experts concur in considering SARS as a different disease in the context of the SARS-CoV2 infection. So, back to the question in the header, what kind of treatment are we going to choose to prevent SARS in people at risk, an antiviral, an anti-inflammatory or both?

\section{Antivirals}

Even if we assume that Covid-19 is a two headed disease (a viral infection and a, more or less, independent acute autoimmune disorder), we could easily agree on this: using antivirals would be a good way to start treating the disease. In fact, following this reasonable criteria, antivirals have been profusely used since the very beginning of the pandemic (both in China and in Europe). The peak of antiviral popularity came when the US authorities gave credit to the use of a singled out antiviral (Remdesivir) that at some point was touted by the media as "the" miracle drug against the disease ${ }^{[20][21][22]}$. But, as we said before, since Covid-19 is a two-headed disease, and the one aspect that we want to prevent is not the viral infection itself but the inflammatory reaction that triggers SARS, is it that important to stop viral replication? l'll give you a short answer: it is not that important. But why?

As counterintuitive as it could sound, there are strong reasons to believe that the load of viral infection by SARS-Cov2 (as well as its measurable surrogates, viral load and viremia) is not relevant to the appearance and progression of the lethal complications of the Covid 19 disease. First, a multicenter study published in Nature Medicine (15 April 2020) ${ }^{\text {[23] }}$ concludes that "There was no obvious difference in viral loads across sex, age groups and disease severity,". In another study on hospitalized patients, viral load is similar in mild vs severe cases and in people under or over age $65^{[24]}$. Moreover, people who are asymptomatic all through the infection have the same viral load as patients with covid-19 symptoms ${ }^{[25]}$. Evidence also comes from studies in mice, thus older mice (risk group) infected with the SARS-CoV1 virus, although they present a mild viral infection (i.e. low viral load), they develop a form of lethal SARS similar to the human 
SARS ${ }^{[26]}$. Finally, the lung viral replication kinetics is similar in AC70 and AC22 mice, two lineages representing respectively those susceptible and resistant to the lethal SARS-CoV infection ${ }^{[27]}$.

To recap, viral infection doesn't seem to be the key target if you want to prevent death in Covid-19.

\section{Anti-inflammatories}

For the last 4 months I have proposed and extensively used a treatment that, although still not proven in a formal trial, really prevents death in this disease. The treatment consists in using (in patients at risk) an inhaled corticosteroid (budesonide) together with a long acting beta agonist (formoterol). The rationale for the treatment is written somewhere else so I won't expand much on that in here ${ }^{[28]}$. During asthma attacks, this treatment is recommended to be administered on an "as needed" dosage schedule, so we will refer to it henceforth as "As Needed Inhaled Budesonide/Formoterol" or ANIBF. Back in March/April, I was studying the apparent correlation of asthma \& allergic diseases with a better outcome in the Covid-19 disease, and I was then pondering that perhaps they, asthmatic patients in particular, were protected from Covid-19, at least in part, because of the chronic preventive IBF schedule many of them were following on a chronic basis (I realized later that the picture was a little bit more complex than that ${ }^{[29]}$ ). As a general pediatrician, my understanding was that inhalers in asthma, first of all, are meant to provide a background bronchodilation tone (the reason for using a long acting bronchodilator like formoterol) plus a mild local immune suppressor effect (accomplished by budesonide). But, after digging deeper into the molecular underpinnings of the IBF combination, I soon realized that the picture was much more complex than that, and that the actual end effect of IBF was more that of a compound drug with both potential antiviral and strong anti-inflammatory powers. That budesonide is an immunosuppressant, we knew it already, but formoterol? Formoterol, on the other hand, has been used in asthma as a bronchodilator, not as an anti-inflammatory. As it turns out, formoterol alone can be either pro or anti-inflammatory, but, and this is the important part, when combined with an ICS (budesonide in this case), it invariably synergizes to decrease inflammation. Much more than budesonide alone!

\section{An off-label treatment for Covid-19}

For those not familiar with the term, this is what Wikipedia says: "Off-label use is the use of pharmaceutical drugs for an unapproved indication or in an unapproved age group, dosage, or route of administration. Both prescription drugs and over-the-counter drugs (OTCs) can be used in off-label ways, although most studies of off-label use focus on prescription drugs. Off-label use is generally legal unless it violates ethical guidelines or safety regulations." So, would we violate any ethical guidlines or safety regulations by giving ANIBF to patients at risk of dying of SARS? As we will see in the coming pages, surely not. Rather, the opposite.

We will not argue anymore on whether CS, in general, or budesonide, in particular, are antiviral or not, since, as we already know, viral load is not the relevant problem in SARS. Inflammation is. But, in case there is some legitimate concern, we will show next that the combination of budesonide/formoterol, besides being a potent anti-inflammatory compound (for a review on the antiinflammatory effects of IBF see for reviews on the subject ${ }^{[30][31]}$ ) is also a bona fide very efficient antiviral drug. 
And this is to a great extent due to the fact that formoterol is a potent antiviral itself. What? Indeed, in a screening of 1500 off-patent SMD, only four compounds stood out to repress coxsackie virus replication. One of them, surprise, was formoterol, probably inhibiting the genome replication phase of the virus ${ }^{[32]}$. In fact, as it turns out, formoterol is a broad inhibitor of enteroviruses ${ }^{[33]}$. Also, elsewhere, mice infected with the human influenza virus developed pulmonary edema that was abrogated by treatment with formoterol ${ }^{[34]}$. In another study, formoterol, was able to prevent the acute effects of sepsis induced by liposaccharide (LPS) injection on rat gastrocnemius muscle ${ }^{[35]}$. Again, in a compound screening of FDA approved drugs, formoterol, guess what, was found to bind with significant affinity to the papain-like protease (PLpro) of SARS-CoV2 (yes, the Cov-19 virus!) ${ }^{[36]}$. Finally, in case you were still worried by viral loads, formoterol alone showed a very robust inhibition of replication of the SARS-CoV2 cousin, the coronavirus HCoV-229E ${ }^{[37]}$. Finally adding budesonide to formoterol, not only does not counter the antiviral effect of formoterol, but, unsurprisingly, have an additive antiviral

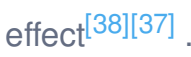

To summarize, IBF is both anti-viral and, most importantly, a strong antiinflammatory drug, with an immaculate safety track and it should be used as early as possible (even without a positive Covid-19 test), as soon as the disease is suspected in people at risk of developing SARS.

So, you may ask, what are we waiting to give this potentially silver bullet drug to people at risk of dying from Covid-19? A drug that millions of asthmatic people (many of them Covid-19+) are saying is safe and effective.

\section{ANIBF as an off-label indication for Covid-19 in patients at risk of developing SARS}

In more detail, here is therefore my ranking of favorites to do the job of preventing death in Covid-19:

1. Inhaled Budesonide+Formoterol (ANIBF) as a de facto off-label medication for Covid-19. By far, on top of the list. This combination has every chance to do the job better than anything else: First, it is inhaled (i.e. locally applied where the fire ignites, in the lower airways). Second, it is antiviral and (more importantly) strongly anti-inflammatory. Third, and perhaps the most important one at this stage of the pandemic, it is SAFE. It is safe even in the context of the Covid19 infection. Just to illustrate the latter point, has anybody heard of the potential hazard of using IBF in covid-19 infected people (as millions of people around the world do everyday for their asthma)? The fact that asthmatic people on IBF are fighting Covid-19 better than other people of the same age and health preconditions, should be enough to start recommending IBF to everybody at risk of dying from the disease. My personal recommendation: if you are older, live in a nursing home, are diabetic, obese or hypertense, or have any other Covid-19 risk comorbidity, don't wait until the FDA, or your local Drug Agency gives you the approval. Remember, this is an off-label indication for non-asthmatic people with suspected or confirmed Covid-19. You only need to fill two requirements: being considered at risk of dying from Covid-19 and having your doctor's prescription (the same that you need for an antibiotic or an anti-depressant). So, talk to your doctor, get the inhaler (IBF $320 / 9 \mathrm{mcg}$ ), and put it on a visible place on your bedside table. The rest will 
be easy-peasy. As soon as you start having suspicious symptoms (cough, diarrhea, anosmia, headache, etc), even without a positive test yet, begin with a couple of puffs every 8 hours and increase the frequency as needed in the ensuing days. Very important, don't wait for the ominous sign (dyspnea) to start with the treatment. If you follow my advice, you will feel much better as days, weeks go by. Keep adjusting the dose to your symptoms and do this for a minimum of a month, even if you feel that everything is gone. Just believe me! Oh, and let your doctor monitor your treatment all along.

2. Any combination of formoterol with other ICS like fluticasone or beclomethasone (It would take a separate paper to argue that formoterol is largely preferred to another LABA, salmeterol[39][40][41]). Remember, don't use Beta agonists alone (either long acting or short acting like salbutamol/albuterol). Always combine them with an ICS.

3. Inhaled ciclesonide (another corticosteroid!). Ciclesonide is antiviral in vitro. In a screen of a library of approved drugs, searching for anti-SARS-CoV-2, ciclesonide stood out with only one other compound, as an inhibitor of viral replication ${ }^{[42]}$, apparently by inhibiting the function of NSP15, a protein involved in impairing the recognition of viral dsRNA by host sensors, thus escaping host vigilance ${ }^{[43]}$. Also, ciclesonide clinically improves the outcome of Covid-19 disease in the small number of patients ${ }^{[44]}$. By the way, which do you think is the inhaled corticosteroid structurally closest to ciclesonide? If your answer was budesonide, you were right To recap on ciclesonide, I give you three reasons to consider it as a good treatment alternative: a) it is a local treatment (remember the real problem is in the lungs, where the fire ignites); b) is a corticosteroid, and c) they use it in Japan (a country that has been notorious for its comparatively very mild version of the pandemic) and my guess is that ciclesonide has been being used during the pandemic in Japanese primary care facilities as well as medical wards more that it has been publicly admitted. You draw your own conclusions.

4. Inhaled corticosteroid (any) alone. If you can't afford an IBF or if it is not available at your place, you can use any ICS. The preferred one: budesonide. Use it on an "as needed" schedule under your doctor's supervision.

5. Inhaled heparin. Not for the reasons you think. Inhaled heparin is safe and has no systemic side-effects ${ }^{[45]}$. It has been shown to possess broad airway anti-inflammatory activity on different models of airway inflammation ${ }^{[46][47]}$ and the use of prophylactic low molecular weight heparin in non-ICU patients is associated with a reduced heparanase activity and therefore decreased inflammation ${ }^{[48]}$. Importantly, Inhaled heparin has been used to treat Acute Respiratory Distress Syndrome from other causes ${ }^{[49][50]}$ and also as a broad indication for lung injury ${ }^{[51][52]}$. Finally, for those still concerned with viral loads, not surprisingly[53][54][55][56], heparin inhibits the binding of SARS-CoV-2 spike protein to human cell lines ${ }^{[57]}$.

I could mention several additional alternatives but, for the sake of keeping the list of true candidates as short as possible, I will stop here.

To summarize, nobody can say anymore that there is no treatment for Covid-19. Here is one that is both an antiviral and a very strong anti inflammatory. It comes as an inhaler, something very important, since it has a direct effect on the epicenter of SARS, the lungs, and also because it forfends the side effects associated with the use of systemic drugs. I'm pretty sure that, by preventing SARS, in most Covid-19 cases, we won't need any assisted ventilation, anticoagulation, vasoactive 
drugs, expensive humanized antibodies, fancy iRNAs, or any other drug aimed at alleviating Covid-19 patients once in the ICU. Instead, we will be preventing people from having to attend hospital ERs or being admitted to wards, let alone to ICUs. This is a disease we should be able to handle at home, with the simple means of a finger pulse oximeter, an IBF inhaler, and the watchful monitoring by your family doctor.

\section{References}

1. ^Simon B. Drysdale, Rachael S. Barr, Christine S. Rollier, Christopher A. Green, Andrew J. Pollard, Charles J. Sande. (2020). Priorities for developing respiratory syncytial virus vaccines in different target populations. Sci. Transl. Med., vol. 12 (535), eaax2466. doi:10.1126/scitrans/med.aax2466.

2. `Muhammad Shafique, Muhammad Asif Zahoor, Muhammad Imran Arshad, Bilal Aslam, Abu Baker Siddique, Muhammad Hidayat Rasool. (2019). Hurdles in Vaccine Development against Respiratory Syncytial Virus. doi:10.5772/intechopen.87126.

3. ' Jason A. Tetro. (2020). Is COVID-19 receiving ADE from other coronaviruses?. Microbes and Infection, vol. 22 (2), 7273. doi:10.1016/j.micinf.2020.02.006.

4. ' Akiko Iwasaki, Yexin Yang. (2020). The potential danger of suboptimal antibody responses in COVID-19. Nat Rev Immunol, vol. 20 (6), 339-341. doi:10.1038/s41577-020-0321-6.

5. ^ Peter J. Hotez, David B. Corry, Maria Elena Bottazzi. (2020). COVID-19 vaccine design: the Janus face of immune enhancement. Nat Rev Immunol, vol. 20 (6), 347-348. doi:10.1038/s41577-020-0323-4.

6. ' Carlos Rodriguez. (2020). Good news from Alsace: the Covid-19 pandemic is (almost) over. NOW WHAT?. Qeios. doi:10.32388/uo6rt1.2.

7. ^ Haley E. Randolph, Luis B. Barreiro. (2020). Herd Immunity: Understanding COVID-19. Immunity, vol. 52 (5), 737-741. doi:10.1016/j.immuni.2020.04.012.

8. ^ Tom Britton, Pieter Trapman, Frank G Ball. (2020). The disease-induced herd immunity level for Covid-19 is substantially lower than the classical herd immunity level. doi:10.1101/2020.05.06.20093336.

9. `Tanishque Propkar Malik. (2020). Modelling and Analysis of 2019-nCov Epidemic in India : A study on Attack \&amp: Recovery Rate, Basic \&amp; Effective Reproductive Number, Herd Immunity Threshold \&amp; Case Fatality Ratio of Coronavirus Covid-19 in India.. doi:10.35543/osf.io/cwyeu.

10. ^ Floriane Gallais, Aurelie Velay, Marie-Josee Wendling, Charlotte Nazon, Marialuisa Partisani, Jean Sibilia. (2020). Intrafamilial Exposure to SARS-CoV-2 Induces Cellular Immune Response without Seroconversion. doi:10.1101/2020.06.21.20132449.

11. ^ Nina Le Bert, Anthony T. Tan, Kamini Kunasegaran, Christine Y. L. Tham, Morteza Hafezi, Adeline Chia. (2020). 
SARS-CoV-2-specific T cell immunity in cases of COVID-19 and SARS, and uninfected controls. Nature. doi:10.1038/s41586-020-2550-z.

12. 'Yukihiko Nakata. (2010). Global dynamics of cell mediated immunity in viral infection models with distributed delays.

13. ^ Elizabeth Krieger, Nicole Vissichelli, Stefan Leichtle, Markos Kashioris, Roy Sabo, Don Brophy. (2020). Immunological determinants of clinical outcomes in COVID-19: A quantitative perspective.

14. ^ M. G. Netea, L. A. B. Joosten, E. Latz, K. H. G. Mills, G. Natoli, H. G. Stunnenberg. (2016). Trained immunity: A program of innate immune memory in health and disease. Science, vol. 352 (6284), aaf1098-aaf1098. doi:10.1126/science.aaf1098.

15. ^Anthony Harries, Kudakwashe C Takarinda. (2020). Faculty Opinions recommendation of Presenting Characteristics. Comorbidities, and Outcomes Among 5700 Patients Hospitalized With COVID-19 in the New York City Area.. doi:10.3410/f.737797860.793574198.

16. ^ Adekunle Sanyaolu, Chuku Okorie, Aleksandra Marinkovic, Risha Patidar, Kokab Younis, Priyank Desai. (2020). Comorbidity and its Impact on Patients with COVID-19. SN Compr. Clin. Med.. doi:10.1007/s42399-020-00363-4.

17. ^ Luis F. García. (2020). Immune Response, Inflammation, and the Clinical Spectrum of COVID-19. Front. Immunol., vol. 11. doi:10.3389/fimmu.2020.01441.

18. ^ Alice Huertas, David Montani, Laurent Savale, Jérémie Pichon, Ly Tu, Florence Parent. (2020). Endothelial cell dysfunction: a major player in SARS-CoV-2 infection (COVID-19)?. Eur Respir J, vol. 56 (1), 2001634. doi:10.1183/13993003.01634-2020.

19. ^ (2020). Pulmonary Vascular Pathology in Covid-19. N Engl J Med. doi:10.1056/nejmc2022068.

20. ^ Jonathan Grein, Norio Ohmagari, Daniel Shin, George Diaz, Erika Asperges, Antonella Castagna. (2020). Compassionate Use of Remdesivir for Patients with Severe Covid-19. N Engl J Med, vol. 382 (24), 2327-2336. doi:10.1056/nejmoa2007016.

21. ^ Robin E Ferner, Jeffrey K Aronson. (2020). Remdesivir in covid-19. BMJ. doi:10.1136/bmj.m1610.

22. ^ Peter M. Goodwin. (2020). Small Study Finds Remdesivir Benefit in COVID-19 But Questions Remain. Oncology Times, vol. 42 (10), 28-29. doi:10.1097/01.cot.0000668244.87235.a5.

23. ^ Xi He, Eric H. Y. Lau, Peng Wu, Xilong Deng, Jian Wang, Xinxin Hao. (2020). Temporal dynamics in viral shedding and transmissibility of COVID-19. Nat Med, vol. 26 (5), 672-675. doi:10.1038/s41591-020-0869-5.

24. ` Yu Chen, Lanjuan Li. (2020). SARS-CoV-2: virus dynamics and host response. The Lancet Infectious Diseases, vol. 20 (5), 515-516. doi:10.1016/s1473-3099(20)30235-8.

25. ^ D Cereda, M Tirani, F Rovida, V Demicheli, M Ajelli, P Poletti. (2020). The early phase of the COVID-19 outbreak in Lombardy, Italy.

26. ^ Jun Chen, Yuk Fai Lau, Elaine W. Lamirande, Christopher D. Paddock, Jeanine H. Bartlett, Sherif R. Zaki. (2009). Cellular Immune Responses to Severe Acute Respiratory Syndrome Coronavirus (SARS-CoV) Infection in Senescent BALB/c Mice: CD4+ T Cells Are Important in Control of SARS-CoV Infection. JVI, vol. 84 (3), 1289-1301. doi:10.1128/jvi.01281-09.

27. ^ Naoko Yoshikawa, Tomoki Yoshikawa, Terence Hill, Cheng Huang, Douglas M. Watts, Shinji Makino. (2009). Differential Virological and Immunological Outcome of Severe Acute Respiratory Syndrome Coronavirus Infection in 
Susceptible and Resistant Transgenic Mice Expressing Human Angiotensin-Converting Enzyme 2. JVI, vol. 83 (11), 5451-5465. doi:10.1128/jvi.02272-08.

28. ` K. R. Chapman, N. C. Barnes, A. P. Greening, P. W. Jones, S. Pedersen. (2010). Single maintenance and reliever therapy (SMART) of asthma: a critical appraisal. Thorax, vol. 65 (8), 747-752. doi:10.1136/thx.2009.128504.

29. ^ Carlos Rodriguez, Carlos Veciana. (2020). ASTHMA AND COVID-19: THE EOSINOPHILIC LINK. Qeios. doi:10.32388/5iy4if.

30. ^ C. L. Skevaki, I. Christodoulou, I. S. Spyridaki, I. Tiniakou, V. Georgiou, P. Xepapadaki. (2009). Budesonide and formoterol inhibit inflammatory mediator production by bronchial epithelial cells infected with rhinovirus. doi:10.1111/j.1365-2222.2009.03307.x.

31. ` THE COMBINATION OF INHALED BUDESONIDE AND FORMOTEROL AS AN EARLY TREATMENT FOR THE COVID-19 DISEASE. zenod.

32. ^ Rachel Ulferts, S. Matthijn de Boer, Lonneke van der Linden, Lisa Bauer, Hey Rhyoung Lyoo, Maria J. Maté. (2016). Screening of a Library of FDA-Approved Drugs Identifies Several Enterovirus Replication Inhibitors That Target Viral Protein 2C. Antimicrob. Agents Chemother., vol. 60 (5), 2627-2638. doi:10.1128/aac.02182-15.

33. ^ Els Wessels, Daniël Duijsings, Richard A. Notebaart, Willem J. G. Melchers, Frank J. M. van Kuppeveld. (2005). $\underline{A}$ Proline-Rich Region in the Coxsackievirus 3 A Protein Is Required for the Protein To Inhibit Endoplasmic Reticulum-toGolgi Transport. JVI, vol. 79 (8), 5163-5173. doi:10.1128/jvi.79.8.5163-5173.2005.

34. ^ Susan M. Armstrong, Changsen Wang, Jayesh Tigdi, Xiaoe Si, Carlo Dumpit, Steffany Charles. (2012). Influenza Infects Lung Microvascular Endothelium Leading to Microvascular Leak: Role of Apoptosis and Claudin-5. PLOS ONE, vol. 7 (10), e47323. doi:10.1371/journal.pone.0047323.

35. ^Ana Isabel Martín, Ana Belén Gómez-SanMiguel, Teresa Priego, Asunción López-Calderón. (2018). Formoterol treatment prevents the effects of endotoxin on muscle TNF/NF-kB, Akt/mTOR, and proteolytic pathways in a rat model. Role of IGF-I and miRNA 29b. American Journal of Physiology-Endocrinology and Metabolism, vol. 315 (4), E705E714. doi:10.1152/ajpendo.00043.2018.

36. ^ Rimanshee Arya, Amit Das, Vishal Prashar, Mukesh Kumar. (2020). Potential inhibitors against papain-like protease of novel coronavirus (SARS-CoV-2) from FDA approved drugs. doi:10.26434/chemrxiv.11860011.v2.

37. ${ }^{a, b}$ Mutsuo Yamaya, Hidekazu Nishimura, Xue Deng, Mitsuru Sugawara, Oshi Watanabe, Kazuhiro Nomura. (2020).

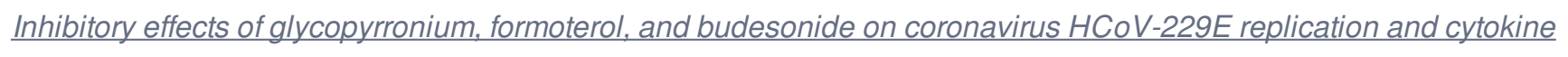
production by primary cultures of human nasal and tracheal epithelial cells. Respiratory Investigation, vol. 58 (3), 155168. doi:10.1016/j.resinv.2019.12.005.

38. ^ Mutsuo Yamaya, Hidekazu Nishimura, Lusamba Nadine, Hiroshi Kubo, Ryoichi Nagatomi. (2014). Formoterol and budesonide inhibit rhinovirus infection and cytokine production in primary cultures of human tracheal epithelial cells. Respiratory Investigation, vol. 52 (4), 251-260. doi:10.1016/j.resinv.2014.03.004.

39. ^ Karin Strandberg, Lena Palmberg, Kjell Larsson. (2007). Effect of formoterol and salmeterol on IL-6 and IL-8 release in airway epithelial cells. Respiratory Medicine, vol. 101 (6), 1132-1139. doi:10.1016/j.rmed.2006.11.014.

40. ^ L. E. Donnelly, S. J. Tudhope, P. S. Fenwick, P. J. Barnes. (2009). Effects of formoterol and salmeterol on cytokine release from monocyte-derived macrophages. European Respiratory Journal, vol. 36 (1), 178-186. 
doi:10.1183/09031936.00158008.

41. ^ Gregory R. Tintinger, Annette J. Theron, Helen C. Steel, Charles Feldman, Ronald Anderson. (2015). Formoterol is more effective than salmeterol in suppressing neutrophil reactivity. ERJ Open Res, vol. 1 (1), 00014-2015. doi:10.1183/23120541.00014-2015.

42. `Sangeun Jeon, Meehyun Ko, Jihye Lee, Inhee Choi, Soo Young Byun, Soonju Park. (2020). Identification of Antiviral Drug Candidates against SARS-CoV-2 from FDA-Approved Drugs. Antimicrob Agents Chemother, vol. 64 (7). doi:10.1128/aac.00819-20.

43. `Xufang Deng, Matthew Hackbart, Robert C. Mettelman, Amornrat O’Brien, Anna M. Mielech, Guanghui Yi. (2017). Coronavirus nonstructural protein 15 mediates evasion of dsRNA sensors and limits apoptosis in macrophages. Proc Natl Acad Sci USA, vol. 114 (21), E4251-E4260. doi:10.1073/pnas.1618310114.

44. `Keisuke Iwabuchi, Koichiro Yoshie, Yuichi Kurakami, Kota Takahashi, Yoshio Kato, Tsuneo Morishima. (2020). Therapeutic potential of ciclesonide inhalation for COVID-19 pneumonia: Report of three cases. Journal of Infection and Chemotherapy, vol. 26 (6), 625-632. doi:10.1016/j.jiac.2020.04.007.

45. ^ K.E. Bendstrup, J. Gram, J.I. Jensen. (2002). Effect of inhaled heparin on lung function and coagulation in healthy volunteers. Eur Respir J, vol. 19 (4), 606-610. doi:10.1183/09031936.02.00105202.

46. ^ISTELMACH. (2000). 578 The effect of inhaled heparin on bronchial hyperresponsiveness measured by histamin challenge. Journal of Allergy and Clinical Immunology, vol. 105 (1), S193. doi:10.1016/s0091-6749(00)91006-6.

47. ^ I. Pavord, T. Mudassar, J. Bennett, P. Wilding, A. Knox. (1996). The effect of inhaled heparin on bronchial reactivity to sodium metabisulphite and methacholine in patients with asthma. doi:10.1183/09031936.96.09020217.

48. ^Baranca Buijsers, Cansu Yanginlar, Inge Grondman, Aline de Nooijer, Marissa L Maciej-Hulme, Inge Jonkman. (2020). Increased plasma heparanase activity in COVID-19 patients. doi:10.1101/2020.06.12.20129304.

49. ^MohamedH Saleh, Emad Omar. (2017). Does nebulized heparin have value in acute respiratory distress syndrome patients in the setting of polytrauma?. Egypt J Bronchol, vol. 11 (4), 332. doi:10.4103/ejb.ejb_24_17.

50. ^Umair Ashraf, Bharat Bajantri, Gabriella Roa-Gomez, Sindhaghatta Venkatram, Amanda Cantin, Gilda Diaz-Fuentes. (2018). Nebulized heparin and N-acetylcysteine for smoke inhalational injury. Medicine, vol. 97 (19), e0638. doi:10.1097/md.0000000000010638.

51. ^Umair Ashraf, Bharat Bajantri, Gabriella Roa-Gomez, Sindhaghatta Venkatram, Amanda Cantin, Gilda Diaz-Fuentes. (2018). Nebulized heparin and N-acetylcysteine for smoke inhalational injury. Medicine, vol. 97 (19), e0638. doi:10.1097/md.0000000000010638.

52. ^Andrew C. Miller, Elamin M. Elamin, Anthony F. Suffredini. (2014). Inhaled Anticoagulation Regimens for the Treatment of Smoke Inhalation-Associated Acute Lung Injury. Critical Care Medicine, vol. 42 (2), $413-419$. doi:10.1097/ccm.0b013e3182a645e5.

53. ^ Chao Gao, Junwei Zeng, Nan Jia, Kathrin Stavenhagen, Yasuyuki Matsumoto, Hua Zhang. (2020). SARS-CoV-2 Spike Protein Interacts with Multiple Innate Immune Receptors. doi:10.1101/2020.07.29.227462.

54. ^So Young Kim, Weihua Jin, Amika Sood, David W. Montgomery, Oliver C. Grant, Mark M. Fuster. (2020). Glycosaminoglycan binding motif at S1/S2 proteolytic cleavage site on spike glycoprotein may facilitate novel coronavirus (SARS-CoV-2) host cell entry. doi:10.1101/2020.04.14.041459. 
55. ^ Paul S. Kwon, Hanseul Oh, Seok-Joon Kwon, Weihua Jin, Fuming Zhang, Keith Fraser. (2020). Sulfated polysaccharides effectively inhibit SARS-CoV-2 in vitro. Cell Discov, vol. 6 (1). doi:10.1038/s41421-020-00192-8.

56. ^ Paolo Maria Matricardi, Roberto Walter Dal Negro, Roberto Nisini. (2020). The first, holistic immunological model of COVID-19: Implications for prevention, diagnosis, and public health measures. Pediatr Allergy Immunol, vol. 31 (5), 454-470. doi:10.1111/pai.13271.

57. ^ Lynda J. Partridge, Lucy Urwin, Martin J.H. Nicklin, David C. James, Luke R. Green, Peter N. Monk. (2020). ACE2independent interaction of SARS-CoV-2 spike protein to human epithelial cells can be inhibited by unfractionated heparin. doi:10.1101/2020.05.21.107870. 